\title{
Simulated Design Strategies for SPECT Collimators to Reduce the Eddy Currents Induced by MRI Gradient Fields
}

\author{
Amine M. Samoudi, Karen Van Audenhaege, Günter Vermeeren, Gregory Verhoyen \\ Luc Martens, Roel Van Holen, and Wout Joseph
}

\begin{abstract}
Combining single photon emission computed tomography (SPECT) with magnetic resonance imaging (MRI) requires the insertion of highly conductive SPECT collimators inside the MRI scanner, resulting in an induced eddy current disturbing the combined system. We reduced the eddy currents due to the insert of a novel tungsten collimator inside transverse and longitudinal gradient coils. The collimator was produced with metal additive manufacturing, that is part of a microSPECT insert for a preclinical SPECT/MRI scanner. We characterized the induced magnetic field due to the gradient field and adapted the collimators to reduce the induced eddy currents. We modeled the $x-, y$-, and $z$-gradient coil and the different collimator designs and simulated them with FEKO, a three-dimensional method of moments / finite element methods (MoM/FEM) full-wave simulation tool. We used a time analysis approach to generate the pulsed magnetic field gradient. Simulation results show that the maximum induced field can be reduced by $50.82 \%$ in the final design bringing the maximum induced magnetic field to less than $2 \%$ of the applied gradient for all the gradient coils. The numerical model was validated with measurements and was proposed as a tool for studying the effect of a SPECT collimator within the MRI gradient coils.
\end{abstract}

Index Terms - MRI; SPECT; Eddy currents; Collimator; Tungsten; Final design.

\section{INTRODUCTION}

$\mathrm{O}$ VER the last decade, multimodal imaging techniques have become more and more important. Functional imaging techniques like Positron Emission Tomography (PET) and Single Positron Emission Computed Tomography (SPECT) are combined with anatomical imaging techniques like Computed Tomography (CT) and Magnetic Resonance Imaging (MRI).

Manuscript received $\mathrm{x} x, \mathrm{x}$; revised $\mathrm{x} x, \mathrm{x}$; accepted $\mathrm{x} x, \mathrm{x}$. Date of publication $\mathrm{x} x, \mathrm{x}$; date of current version $\mathrm{x} \mathrm{x}, \mathrm{x}$.

Grant sponsors: This work was supported by the iMinds SIMRET (SImultaneous Magnetic Resonance imaging and Emission Tomography) project, cofunded by iMinds, a research institute founded by the Flemish Government in 2004, and the companies which cofounded the SIMRET project. Roel Van Holen is funded by the Research Foundation-Flanders (FWO, Belgium) and by Ghent University. Karen Van Audenhaege is funded by the agency for Innovation by Science and Technology-Flanders (IWT, Belgium). The authors are with the Department of Information Technology (INTEC) and the Department of Electronics and Information Systems (ELIS), Ghent UniversityliMinds,B-9000 Ghent, Belgium. Correspondence to: Amine M. Samoudi, E-mail: amine.samoudi@intec.ugent.be
Integrated PET/MR hybrid imaging combines anatomical images with an excellent soft tissue contrast provided by MR with high sensitivity and quantitative molecular images provided by PET. In 2010, the first commercially available whole-body systems for PET/MR hybrid imaging entered the market, based on two separate MR and PET imagers in one [1] followed by a fully integrated whole-body PET/MR hybrid imaging system [2] that enables simultaneous PET/MR data acquisition. Since then, the number of worldwide installations of PET/MR systems has steadily increased [3]. Different groups investigated MR compatibility of the combined PET/MRI systems. Examples of interference phenomena have been reported by several research groups: Wehrl et al. [4] observed image degradation of the MRI due to the presence of a PET detector. Schlyer et al. observed degradation of the PET performance due to the RF pulses and switching gradients of the MRI [5]. Wehner et al. recently presented an MRcompatibility study performed with the world's first preclinical PET/MR insert based on fully digital silicon photo multipliers and they observed an SNR degradation that can be improved by power supply unit shielding [6]. They also found that the PET system works stable even under unrealistic demanding stress tests.

Preclinical SPECT/MRI combines the high-resolution molecular information from SPECT with the excellent soft tissue contrast of MRI, together with localized chemical and physical information such as metabolite concentrations and water diffusion characteristics from MRI. In 2008, Goetz et al. [7] presented the first sequential small animal SPECT/MRI for low field (0.1 T) MRI. Later, Hamamura et al. [8] showed the feasibility of simultaneous SPECT/MRI in a 4T MRI based on a rotating cadmium zinc telluride (CZT) nuclear radiation detector with a parallel hole collimator. Their work uses simplified models and should be considered as a feasibility study. Recently, Cai et al. [9] presented a stationary MR compatible small animal imaging SPECT system based on twenty second-generation energy-resolved photon-counting (ERPC) CdTe detectors. Each detector is associated with four pinholes on average. Another ongoing work for SPECT/MRI is the INSERT (Integrated SPECT/MRI for enhanced Stratification in Radio-chemo Therapy) project [10]. Their final goal is to develop both a clinical brain SPECT insert and a preclinical SPECT insert that can be used inside 
commercially available MRI systems such as a 3T MRI with a 59-cm-bore. Finally, Mediso [11] was first-to-market with a sequential preclinical SPECT/MRI system with a 1-Tesla permanent magnet, $450-\mathrm{mT} / \mathrm{m}$ gradient strength, up to $200 \mathrm{x}$ $250 \mathrm{~mm}$ reconstructed field of view (FOV) and a $275-\mu \mathrm{m}$ spatial resolution for SPECT. However, no commercial system for simultaneous SPECT/MRI has been developed up to now.

While this integration of SPECT/MRI offers numerous advantages and new opportunities, it also presents many technological challenges. One of them is the presence of eddy currents in the collimator due to the pulsed magnetic field gradients $[12,13]$. The undesired magnetic field produced by these eddy currents opposes and distorts the linear gradient fields in the region of interest (ROI), which results in image artifacts [14-16]. Although many approaches have been proposed to minimize the occurrence of eddy currents (active and passive shielding coils [17-19], current pulse preemphasis $[14,16,20]$ ), significant distortions will often remain. This is particularly the case in the presence of highly conductive objects where eddy currents are characterized by long time constants [15]. In the study of the preclinical PET/MRI insert [21], Weissler et al. reported that during long EPI sequences, induced eddy currents resulted in ghosting effects in the MR images: 48 of the 184 EPI images showed ghosting. Additionally, the eddy currents resulted in heating of the PET electronics and consequently in a slight adjustment of its count rates (influencing sensitivity and quantification). A study of the induced eddy currents in collimators for SPECT/MRI [22] showed that the induced magnetic field represents up to $4.7 \%$ of the applied gradient field (for a gradient strength of $500 \mathrm{mT} / \mathrm{m}$ and a rise-time of $0.25 \mathrm{~ms}$ ), which might cause visible distortions in the MRI.

In this paper we investigate how to adapt the collimator design in order to maximally reduce the induced magnetic field due to eddy currents. With the current technology, collimators can be produced by additive manufacturing [23], which gives new degrees of freedom to the design and the material. The novelty of this paper is:

i. The application of a numerical model to study the specific problem of induced eddy currents due to the combination of SPECT (collimators) with MRI scanner for a preclinical SPECT/MRI system.

ii. Optimization of the SPECT collimator to reduce eddy currents by introducing smart design modifications.

\section{METHODS}

\section{A. Simulation platform}

The gradient coils [24] and collimators were modeled with FEKO [25], a three-dimensional electromagnetic simulation platform. FEKO uses the method of moments, which provides full-wave solutions of Maxwell's integral equations in the frequency domain. We also used FEKO provided time analysis functionality, allowing electromagnetic problems to be analyzed in the time domain. The relevant computations were performed in the frequency domain, and fast Fourier transform algorithms were used to transform the data to the time domain. First, we extracted frequency components of the gradient pulse by applying fast Fourier transform; we then performed a broadband simulation covering the frequency range of the gradient shape. The output was then post processed to extract the time response of the system, based on the gradient pulse. The setup was simulated with a broadband simulation from 0 to $10 \mathrm{kHz}$ to cover the frequency range of a sinusoidal pulse with a ramp-down time of $0.25 \mathrm{~ms}$.

We performed simulations with $\mathrm{x}-$, $\mathrm{y}$-, and z-gradient coils for preclinical systems (Fig. 1). The coils was fed with 167.24 A and 162.33 A for transverse and longitudinal gradient coils, respectively. The gradient strength was 500 $\mathrm{mT} / \mathrm{m}$, and the maximum gradient deviation in a sphere of $30 \mathrm{~mm}$ was $2.06 \%$ and $0.86 \%$ for transverse and longitudinal gradient coils, respectively.

The validation of the simulation model with measurements was reported in the paper of Samoudi et al. [22]. To validate simulations with the configuration of measurements, they considered a z-gradient coil, a representation of the phantom, a radio-frequency (RF) coil and a cuboid representing the lead block $(\mathrm{Pb})$. The results of the simulations were compared to measurements using a 7T MRI scanner (Bruker Pharmascan). The z-gradient coil and the simulation tool used in [22] are identical to those used in our study. The maximum variation between measurement and simulation was less than $1 \%$ of the applied gradient field $(\mathrm{G}=500 \mathrm{mT} / \mathrm{m})$.

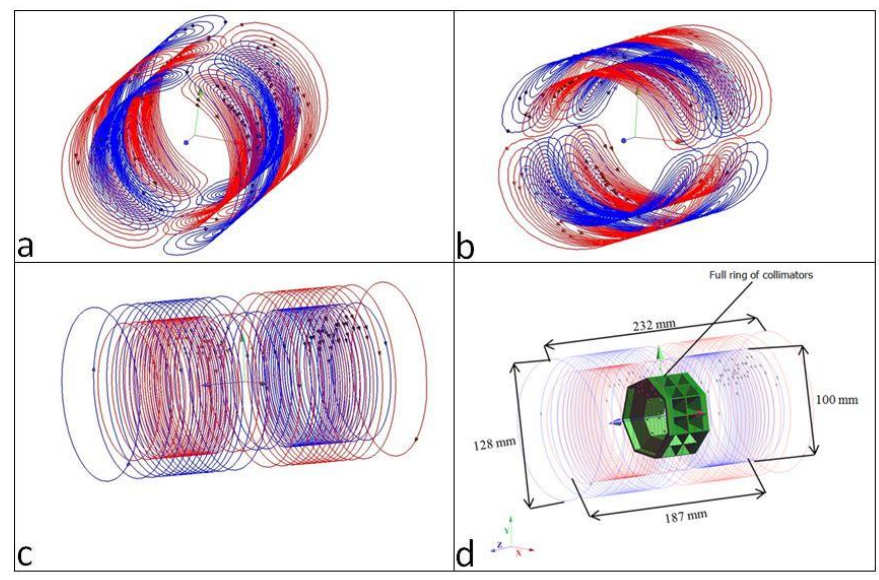

Figure 1: Wire patterns for (a) X-gradient coil. (b) Y-gradient coil and(c) Zgradient coil. (d) Full ring of the collimators centered inside the z-gradient coil. Red and blue colors are used to indicate wires in which there is a different sense of current flow.
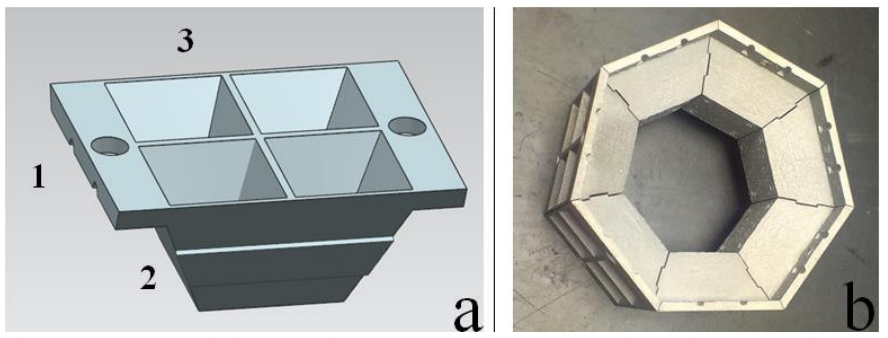

Figure 2: The original collimator. a) $3 \mathrm{D} \mathrm{CAD}$ design of one collimator (1: flange, 2: slope, 3: region of pinholes). b) Printed full-ring multilofthole collimator. 

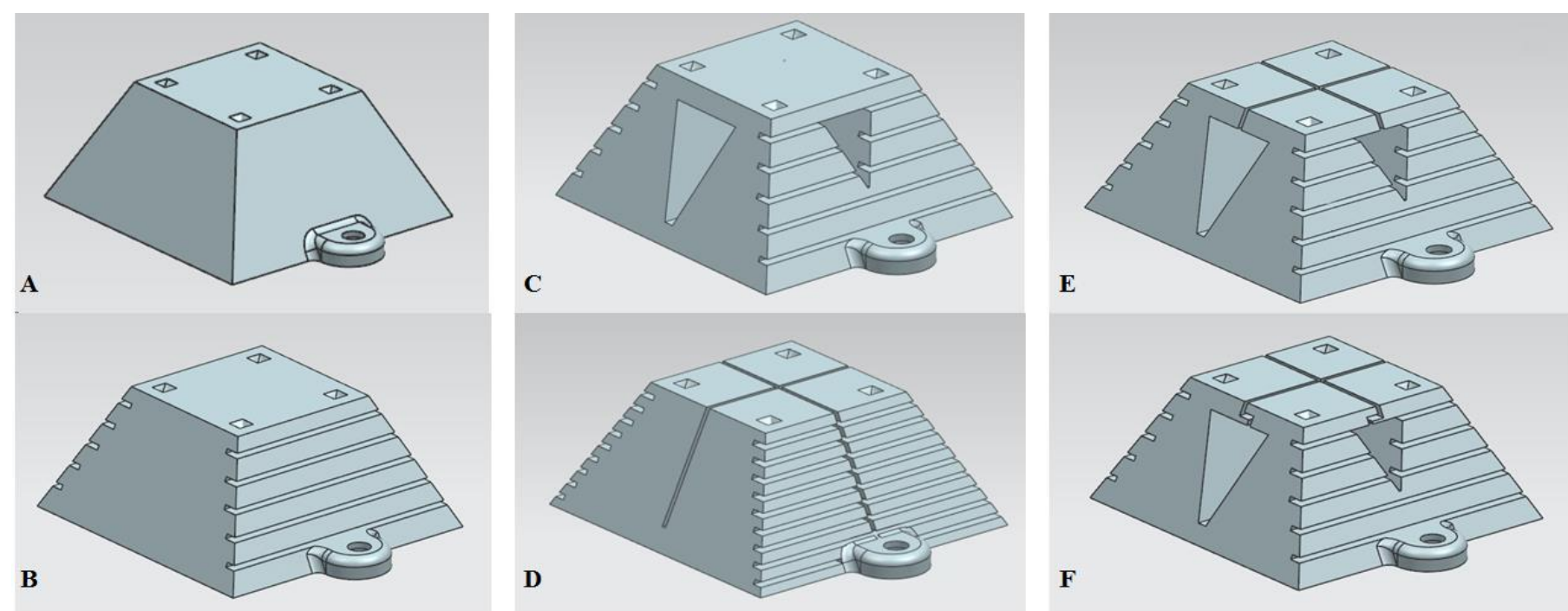

Figure 3: Adapted collimator designs. A): Smaller flanges. B): Horizontal stilts. C): Material reduction in the core. D): Vertical slits in the middle. E): Combined vertical slits and material reduction. F): Z-shaped vertical slit.

\section{B. Original collimator}

Figure 2a shows the proposed collimator that has been developed for a new microSPECT system (Fig. 2b). The system consists of 7 identical collimators and digital silicon photomultipliers assembled in a ring. The system is stationary and therefore less sensitive to geometric calibration issues, better suited for dynamic and gated imaging and easier to integrate with MRI [26, 27].

Tungsten is a promising material for the production of MRcompatible collimators. It has a high number of electrons $(\mathrm{Z}=74)$ and a high density $(19.25 \mathrm{~g} / \mathrm{cm} 3)$. However, pure tungsten is difficult to process. It is both hard and brittle. Therefore, many pinhole collimators are made from tungsten alloys (with nickel, iron and/or copper). They are easier to process but have a higher susceptibility and are thus less MRcompatible. Additive manufacturing overcomes these problems by selective laser melting of pure tungsten powder. This technique can produce complex parts, given some restrictions like a minimum feature size of $500 \mu \mathrm{m}$, a precision of $50 \mu \mathrm{m}$ and a maximum build volume of $245 \mathrm{~mm}$ x $245 \mathrm{~mm}$ x $190 \mathrm{~mm}$ and larger parts will be possible in the future. Down facing surfaces are also difficult to construct or need a supporting structure (Layerwise, Belgium).

Selective laser melting also allows varying the density of the material. The supplier of the collimator is able to produce densities from 80 to $99 \%$. This is interesting because lower density tungsten has a lower conductivity, resulting in less eddy currents. On the other hand, a lower density also results in a lower attenuation coefficient. We chose for a density of 90\% and according to the Beer-Lambert law, $2 \mathrm{~mm}$ of this material is sufficient to attenuate photons with energy of $140 \mathrm{keV}$ which stops $99.85 \%$ of all incoming gamma rays. Due to the additive manufacturing process, the collimator has a different resistivity along the transversal $(292 \mathrm{n} \Omega . \mathrm{m})$ and the longitudinal (108 $\mathrm{n} \Omega . \mathrm{m})$ direction. Because eddy currents increase with the material conductivity, we performed our simulations with a collimator using the lowest resistivity (108 n $\Omega . m)$ to cover the strongest eddy currents.

\section{System optimization}

In order to reduce eddy currents in the collimator ring, we modified the collimator design. We did this step-by-step and investigated the effect of every modification separately. The magnetic field due to eddy currents $\left(\mathrm{B}_{\mathrm{i}}\right)$ was calculated by subtracting the $\mathrm{z}$-component of the magnetic induction of the gradient coils without collimator $\left(\mathrm{B}_{\mathrm{z}}\right)$ from the $\mathrm{z}$-component of the magnetic induction of the gradient coils with the collimators $\left(\mathrm{B}_{\mathrm{z}}^{\mathrm{col}}\right)$. The choice of the $\mathrm{z}$ componenent is motivated by the fact that it represents the main component for the gradient field.

Figure 3 shows the different designs we simulated to reduce the induced magnetic filed due to eddy currents:

\section{1) Smaller flanges}

First, we removed all excess material. The flanges (region 1 in Fig. 2) at the edges of the collimator have as only function to mount the collimator and not to attenuate photons. Therefore, the first adaptation to the design was a reduction of the excessive material in the flanges where high current densities are expected. Proper mounting of the collimator is still possible (Fig. 3A).

\section{2) Horizontal slits}

It is expected that the current density is highest near the surface of the collimator due to the skin effect. This can be reduced with slits in the surface [28, 29]. The distance between the slits is $2 \mathrm{~mm}$, this is necessary not to disturb the function of the collimator [23] (Fig. 3B).

\section{3) Material reduction in the core}

In the center of the collimator there is a solid core of tungsten. The photons are attenuated before they reach the core so this excessive material may be omitted (Fig. 3C). We expect this to further reduce eddy currents but the collimator will be more complex to print and a supporting structure will be needed.

\section{4) Vertical slit in the middle}

The supporting structure can be avoided by using two vertical slits in the middle of the collimator (Fig. 3D) to 
reduce eddy currents instead of removing all the core material.

5) Z-shaped vertical slit

In order to maximally reduce the eddy current, the two previous solutions (sections C.3 and C.4) can be combined (Fig. 3E). However, when the reduction of material in the core is combined with the vertical slits, some photons might not be attenuated and pass though the vertical slits and the thin wall of the lofthole. This penetration can be avoided by using a Z-shaped slit (Fig. 3F and Fig. 4) instead of the vertical slit of Fig. 3D.

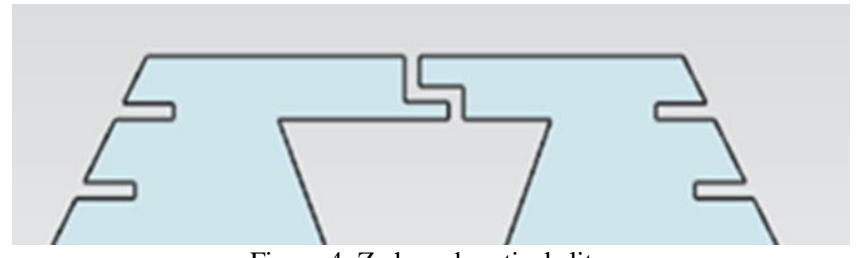

Figure 4: Z-shaped vertical slit.

\section{RESULTS}

\section{A. Current density on the surface of the collimator}

Figure 5 shows current density on the surface of the collimator for different adapted designs shown in Fig. 3, and a comparison between the original and the optimized design (Fig. 5O and 5F) shows a general decrease in current density.

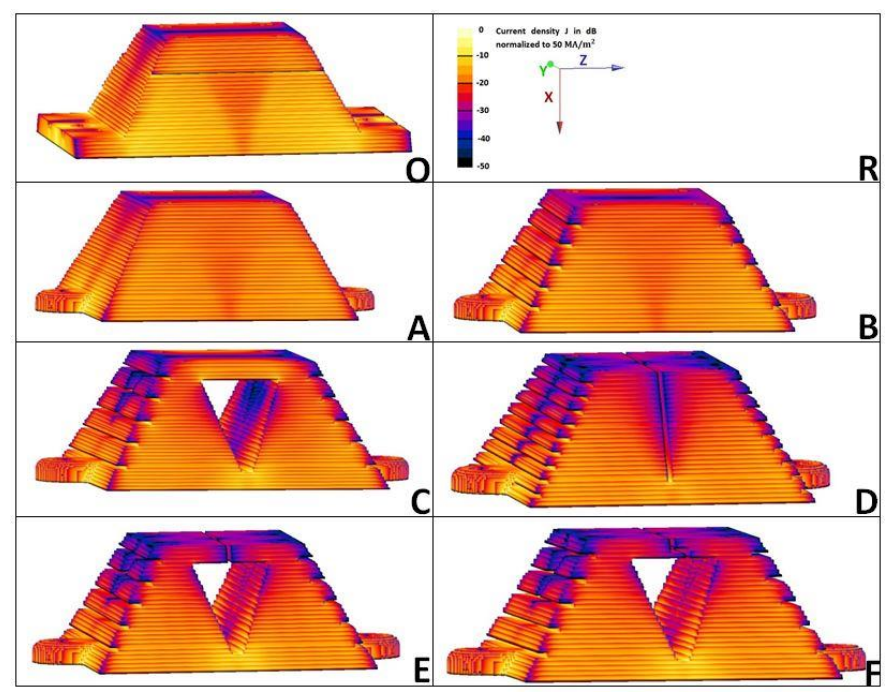

Figure 5: Current density on the surface of a single collimator (RMS value in $\mathrm{dB}$ normalized to $50 \mathrm{MA} / \mathbf{m}^{2}$ ). O: Original collimator as shown in Fig. 2a. $\mathrm{R}$ : Reference and scaling for all the sub-figures. A-F: Adapted collimator designs as shown in Fig. 3.

\section{B. System optimization results}

Table I shows the reduction in the maximum value of the induced magnetic field due to eddy currents inside a FOV of 3 $\mathrm{cm}$, for each adaptation separately for the full ring of 7 collimators. Results show that reduction for collimators inside $\mathrm{x}$ - and $\mathrm{y}$-gradient coil is almost the same (y-gradient coil was designed by simple rotation of the $\mathrm{x}$-gradient coil around the $\mathrm{z}$ axis). We refer to both of the coils as transverse coils. Smaller flanges reduce the current density by $18 \%$ and $16 \%$ for longitudinal and transverse coils ( $\mathrm{x}$ - and $\mathrm{y}$-gradient coils), respectively. The horizontal slits reduce the eddy currents by
$5 \%$ and $4 \%$ for longitudinal and transverse coils, respectively. Using less material in the core or applying vertical slits results in the same reduction of current density. However, the vertical slits are cheaper because a hollow collimator requires supporting structures during production. Both can be combined if z-shaped slits are used to prevent attenuation problems. The reduction is then $26 \%$ and $22 \%$ for longitudinal and transverse coils, respectively. Finally, when all previous adaptations are combined (Fig. 3F), the reduction in eddy currents is about $50.8 \%$ and $43.2 \%$ for longitudinal and transverse coils, respectively.

TABLE I

RESULTS FOR THEDIEFERENT ADAPTATIONS IN THE GRADIENT COILS (EIG. 3

\begin{tabular}{cccc}
\hline Adaptation & Description & Reduction (\%) & Reduction (\%) \\
& for & for
\end{tabular}

longitudinal coil transverse coils

\begin{tabular}{llcc} 
A & Smaller flanges & 18.36 & 15.93 \\
$\mathrm{~B}$ & Horizontal slits $(2 \mathrm{~mm})$ & 5.12 & 4.29 \\
$\mathrm{C}$ & Less material in the & 18.98 & 17.03 \\
& core & 18.75 & 17.21 \\
$\mathrm{D}$ & Vertical slits & 25.23 & 21.81 \\
$\mathrm{E}$ & $\begin{array}{l}\text { Vertical slits and less } \\
\text { material in the core }\end{array}$ & 26.46 & 22.36 \\
$\mathrm{~F}$ & $\begin{array}{l}\text { Vertical z-shaped slits } \\
\text { and less material in the } \\
\text { core }\end{array}$ & & \\
& & \\
\hline
\end{tabular}

\section{Temporal variation of the induced field for the original and the optimized ring of collimators}

Figure 6 shows the magnetic induction due to eddy currents $\left(\mathrm{B}_{\mathrm{i}}\right)$ as a percentage of the applied gradient field for longitudinal and transverse gradient coils ( $\mathrm{x}$ - and $\mathrm{y}$-gradient coils) for the original (Fig. 2a) and the optimized (Fig. 3F) ring of collimators, averaged in a FOV of $3 \mathrm{~cm}$. For the original collimator, the maximum value of the induced magnetic field is $4.21 \%$ and $1.06 \%$ of the applied gradient field (gradient strength $=500 \mathrm{mT} / \mathrm{m}$ ) for longitudinal and transverse gradient coils, respectively. For the optimized ring of collimators, we have $1.91 \%$ and $0.46 \%$ of the applied gradient field for longitudinal and transverse gradient coils, respectively. The transverse coils induce thus less eddy currents than the longitudinal. The maximum value of the induced magnetic field was reduced to less than $2 \%$ of the applied gradient field for both transverse and longitudinal coils.

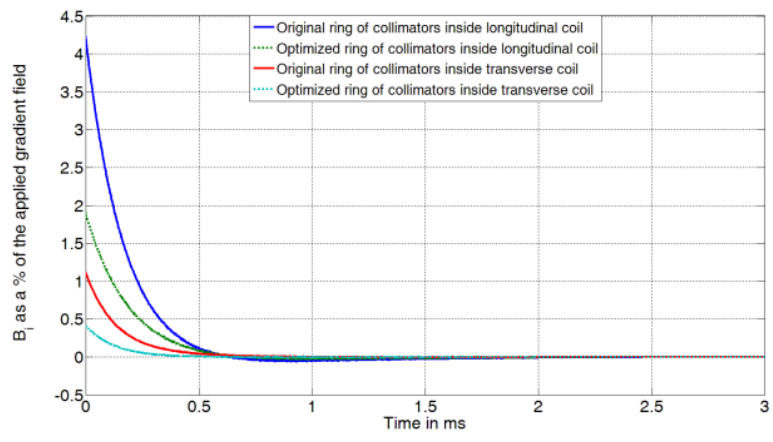

Figure 6: $\mathbf{B}_{\mathbf{i}}$ (induced B-field due to eddy currents) as a percentage of the applied gradient field of $500 \mathrm{mT} / \mathrm{m}$ for both the longitudinal and the transverse gradient coils ( $\mathrm{x}$ - and y-gradient coils) averaged in a FOV of $3 \mathrm{~cm}$. 


\section{DISCUSSION}

Fig. $5 \mathrm{O}$ shows that high values of current density are located in the flanges of the collimators which motivates the first adaptation of our design (smaller flanges).

Table I shows that a high reduction in the maximum induced magnetic field can be obtained. The most important reductions are near the region of the flanges (smaller flanges design) and the center of the collimators (less material in the core and vertical slits designs). A global reduction of $50.8 \%$ and $43.2 \%$ for longitudinal and transverse coils, respectively, is finally obtained with these design changes, and thus by using the optimized design we can bring the percentage of the induced magnetic field to less than $2 \%$ of the applied gradient field. This guideline value of $2 \%$ is experimentally derived during measurements for the SIMRET project.

The z-shape configuration with reduced material in the core seems to be the most favorable since it gives the highest reduction value. The collimator used in the manuscript was optimized for the microSPECT system (in terms of pinholes) and thus, we opted to modify this multi-pinhole collimator instead of making a new one and optimize it for the microSPECT system. A general rule is to omit all unnecessary material (which also makes the production cheaper when using additive manufacturing) while paying attention not to disturb the basic function of the collimator.

Figure 6 shows that the transverse coils induce less eddy currents than the longitudinal coil. This is due to the wire distribution for the two types of the coils and the collimators' position inside the coils.

Translating gradient field distortions to image artefacts is not straight-forward and depends on the sequence used. In fact the distortions will cause artefacts but its shape depends on the trajectory used in the sequence. We note that, the most relevant configuration parameters of the gradient coils for this study are the gradient strength and the rise time of the pulses. Eddy currents increase when the gradient strength increases and decrease when the slope of the gradient pulse becomes steeper.

In the present study, we assume that both the gradient coils and the collimator are passive structures and simulated them with an electromagnetic simulation toolbox based on linear Maxwell equations. We also assume linearity of the system in the method of extraction of the induced magnetic field due to eddy currents. We also focused strictly on the eddy current induced in the tungsten collimators. The proposed work does not consider the effect of coupling of the gradient and/or RF coils, nor the eddy currents on the cryostat magnet, which leads to some remnant errors. However, these could be accounted for through extensions of the methodology presented here, with an extension of the numerical model.

The impact of eddy currents in the SPECT image is not covered in the present study, which focusses on distortions in the MR-gradient field in the presence of a SPECT collimators. However, these could be accounted for future works. The approach used in this paper can be extended to simulate any gradient coil and any collimator design for the purpose of the integration of SPECT/MRI.

\section{CONCLUSION}

In this paper, we investigated and reduced the induced magnetic field due to eddy currents in a full ring of collimators that has been developed for a SPECT/MRI system. A numerical model of the $\mathrm{x}-, \mathrm{y}-$, and $\mathrm{z}$-gradient coils with different designs of the collimators was studied to investigate eddy currents in the tungsten collimators due to the gradient fields for SPECT/MRI system. Simulations were performed using a 3D electromagnetic simulator with a time analysis tool. We made small modifications to the collimator's design and surface and reduced the maximum induced magnetic field by $51 \%$ which results in an improved MR-compatibility. The numerical model and simulation tool were validated in [22]. We conclude that printed tungsten collimators are suited to be used for combined SPECT/MRI systems. The final design presented in this paper can be produced using the promising technique of metal additive manufacturing.

\section{ACKNOWLEDGMENT}

We would like to thank Michael Poole for his help with the gradient coil design.

We would like to thank Carmen Bouckaert for the collimator drawings and printing.

\section{REFERENCES}

[1] H. Zaidi, N. Ojha, M. Morich, et al. "Design and performance evaluation of a whole-body Ingenuity TF PET-MRI system", Phys Med. Biol., vol. 21, pp. 3091-3106, 2011.

[2] G. Delso, S. Furst, B. Jakoby, et al. "Performance measurements of the Siemens mMR integrated whole-body PET/MR scanner", J. Nucl. Med. vol. 52, pp. 1914-1922, 2011.

[3] H. Quick, "Integrated PET/MR", J. Magn. Reson. Imag., vol. 39, pp. 243-258, 2014.

[4] H. Wehrl, M. Judenhofer, A. Thielscher, P. Martirosian, F. Schick, B. Pichler, "Assessment of MR compatibility of a PET insert developed for simultaneous multi-parametric PET/MR imaging on an animal system operating at 7 T" Magnetic Resonance in Medicine 65 (1) (2011) 269.

[5] D. Schlyer, P. Vaska, D. Tomasi, C. Woody, S.H. Maramraju, S. Southekal, J.-F.Pratte, S. Junnarkar, S. Solis-Najera, S. Krishnamoorthy, A. Kriplani, S. Stoll, "A Simultaneous PET/MRI scanner based on RatCAP in small animals", Nuclear Science Symposium Conference Record, 2007 (NSS '07), IEEE, vol. 5, 2007, pp. 3256-3259.

[6] J. Wehner, B. Weissler, P. Dueppenbecker, P. Gebhardt, D. Schug, W. Ruetten, F. Kiessling, V. Schulz. "PET/MRI insert using digital SiPMs: Investigation of MR-compatibility". Nuclear Instruments and Methods in Physics Research A 734 (2014) 116-121.

[7] Goetz C, Breton E, Choquet P, Israel-Jost V, Constantinesco A, "SPECT Low-Field MRI System for Small-Animal Imaging", J. Nucl. Med., vol. 49, pp. 88-93, 2008.

[8] Hamamura M, Ha S, Roeck W, Muftuler T, Wagenaar D, Meier D, Patt B, Nalcioglu O, "Development of an MR-compatible SPECT system (MRSPECT) for simultaneous data acquisition", Phys. Med. Biol., vol. 2010, no. 55, pp. 1563-1575, 2010.

[9] Cai L, Lai X, Shen Z, Chen CT, Meng LJ, "MRC-SPECT: A sub-500 $\mathrm{mm}$ resolution MR-compatible SPECT system for simultaneous dualmodality study of small animals", Nuclear Instruments and Methods in Physics Research A, vol. 734, pp. 147-151, 2014.

[10] Busca P, et al. "Simulation of the expected performance of INSERT: A new multi-modality SPECT/MRI system for preclinical and clinical imaging”. Nucl Instrum and Methods in Physics Research A, vol. 734, pp. 141-146. 2014.

[11] Mediso Medical Imaging Systems. Available online at www.mediso.com; visited on April 2015.

[12] Samoudi A, Van Audenhaege K, Vermeeren G, Poole M, Martens L, Van Holen R, Joseph W, "Influence of collimator insertion on eddy 
currents for different resistivities of tungsten". In Proc. Intl. Soc. Mag. Reso. Med. 22, 2014, pp. 2824.

[13] Samoudi A, Van Audenhaege K, Vermeeren G, Poole M, Martens L, Van Holen R, Joseph W, "Temporal analysis of Z-Gradient coil eddy currents in tungsten collimator with different resistivities for SPECT/MRI". In Proc. the 3rd PSMR Conference on PET/MR and SPECT/MR. EJNMMI Physics, vol. 1 (suppl 1):A22, 2014.

[14] Morich MA, Lampman DA, Dannels WR, Handler, Goldie FTD, "Exact temporal eddy current compensation in magnetic resonance imaging systems", IEEE, Medical Imaging vol. 7, pp. 247-254, 1988.

[15] Crozier S, Eccles C, Beckey F, Fields J, Doddrell D, "Correction of eddy current-induced B0 shifts by receiver reference phase modulation", J. Magn. Reson. vol. 97, pp. 661-665, 1992.

[16] Jehenson P, Westphal M, Schu N, "Analytical method for the compensation of eddy-current effects induced by pulsed magnetic field gradients in NMR systems", J. Magn. Reson., vol. 90, pp. 264-278, 1990.

[17] Mansfield P, Chapman B, "Active magnetic screening of gradient coils in NMR imaging”, J Magn Reson., vol. 66, pp. 573-576, 1986.

[18] Turner R, Bowley RM, "Passive screening of switched magnetic field gradients", J. Phys. E Sci. Instrum., vol. 19, pp. 876-879, 1986.

[19] Lopez HS, Poole M, S. Crozier S, "Evaluating passively shielded gradient coil configurations for optimal eddy current compensation", J. Phys. D: Appl. Phys., vol. 43, pp. 1-12, 2010.

[20] Glover GH, Pelc NJ, "Method for magnetic field gradient eddy current compensation". US patent No 4,698,591 (Milwaukee, WI, General Electric Company)

[21] Weissler B., Gebhardt P.,Dueppenbecker PM et al. "A digital preclinical PET/MRI insert and initial results", IEEE Transactions on Medical Imaging, doi: 10.1109/TMI.2015.2427993.

[22] Samoudi A, Van Audenhaege K, Vermeeren G, Poole M, Tanghe E, Martens L, Van Holen R, Joseph W, "Analysis of eddy currents induced by transverse and longitudinal gradient coils in different tungsten collimators geometries for SPECT/MRI integration". Magnetic Resonance in Medicine, 2014; in press, doi: 10.1002/mrm.25534.

[23] Deprez K, Vandenberghe S, Audenhaege KV, Vaerenbergh JV, Holen $\mathrm{RV}$, "Rapid additive manufacturing of MR compatible multipinhole collimators with selective laser melting of tungsten powder", Med. Phys., vol. 40, 2013.

[24] Poole M, Bowtell R. "Novel gradient coils designed using a boundary element method". Concepts Magn. Reson. vol. B31, pp. 162-175, 2007.

[25] FEKO, EM Software \& Systems-S.A. (Pty) Ltd. Available online at www.feko.info; visited on April 2015.

[26] Deprez K., Van Holen R., and Vandenberghe S. "The lofthole: A novel shaped pinhole geometry for optimal detector usage without multiplexing and without additional shielding." In IEEE Nuclear Science Symposium and Medical Imaging Conference Record (NSS/MIC), IEEE, pp. 3317-3322, 2011.

[27] Van Holen R., Vandeghinste B., Deprez K., and Vandenberghe S. "Design and performance of a compact and stationary microSPECT system.", Medical physics, vol. 40(11), pp. 112501, 2013.

[28] Bernstein MA, King KF, Zhou X, "Handbook of MRI pulse sequences", Boston: Academic Press, 2004

[29] M. Negrazus, D. George, V. Vrankovic, and M. Werner, "Eddy Current Reduction in Fast Ramped Bending Magnets," IEEE transactions on applied superconductivity, vol. 16, no. 2, june 2006.

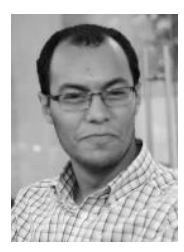

Amine M. Samoudi was born in Oujda, Morocco, on July 20, 1988. He received the Graduate degree in telecoms engineering from the National Institute of Posts and Telecommunications, Rabat, Morocco, in August 2012.

Since February 2013, he has been a member of Wica Research Group with Prof. L. Martens as a Research Engineer at the Department on Information Technology (INTEC), Ghent University, Ghent, Belgium. His research interests include multimodality imaging (SPECT/MR, PET/MR), exposure assessments, and EM Simulations.

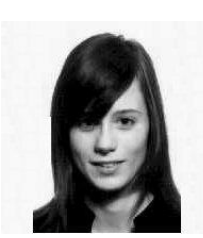

Karen Van Audenhaege obtained a M.S. degree in electrotechnical engineering from the Catholic University of Leuven in 2008. From 2008 to 2010 she worked as a software engineer for Luciad, a Belgian company specialized in geographical information systems and currently, she is pursuing the Ph.D. degree in biomedical engineering at Ghent University. Her research is mainly focused on stationary, MR-compatible brain SPECT imaging based on multi-pinhole collimators.

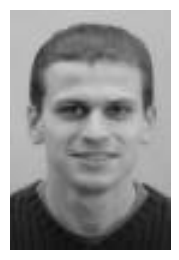

Günter Vermeeren received M. Sc. degree in industrial engineering from the KAHO SintLieven (Ghent, Belgium) in July 1998 and M. Sc. degree in electrical engineering from Ghent University (Belgium) in July 2001.

From September 2001 to September 2002 he joined the research and development department of the network integrator Telindus (Leuven, Belgium). Since September 2002 he was a research engineer in the WiCa group of Prof. Luc Martens. In August 2013, he received the degree of doctor $(\mathrm{PhD})$ in electro-technical engineering at the Ghent University (Belgium). His research focusses on the numerical modelling as well as measurements of electromagnetic radiation in the domain of radio-frequency dosimetry, electromagnetic exposure, on-body propagation and medical imaging systems, such as hybrid MRI systems.

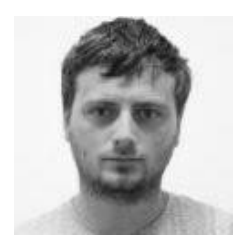

Gregory Verhoyen was born in Roeselare, Belgium, on 7 February. In 2012 he received the Graduate degree in automation and control systems from the University of Leuven, Belgium and in 2014 he received the Graduate degree in Biomedical Engineering from the University of Ghent, Belgium. From august 2014 till January 2015 he has been a member of Medisip Research Group with Prof. R. Vanholen and Wica Research Group with Prof. L. Martens as Research Engineer at the Department on Information Technology (INTEC), Ghent University, Ghent, Belgium. Since January 2015, he is working at Actemium, Aalter, Belgium as Automation engineer, specialty in robotics and motion control. 


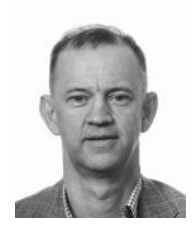

Luc Martens (M'92) received the M.Sc. degree in electrical engineering from the Ghent University, Belgium in July 1986.

From September 1986 to December 1990 he was a research assistant at the Department of Information Technology (INTEC) of the same university. During this period, his scientific work was focused on the physical aspects of hyperthermic cancer therapy. His research work dealt with electromagnetic and thermal modelling and with the development of measurement systems for that application. This work led to the Ph.D. degree in December 1990. Since 1991, he manages the wireless \& cable research group at INTEC. This group is since 2004 part of the iMinds institute and since April 1993 he is Professor at Ghent University. His experience and current interests are in modelling and measurement of electromagnetic channels, of electromagnetic exposure e.g. around telecommunication networks and systems such as cellular base station antennas, and of energy consumption in wireless networks. He is author or co-author of more than 300 publications in the domain of electromagnetic channel predictions, dosimetry, exposure systems and health and wireless communications.

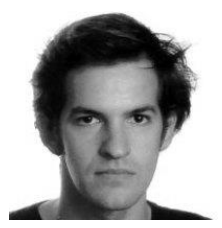

Roel Van Holen (M) is assistant professor at Ghent University. He was born in Halle, Belgium in 1979 and got his Electronics Engineering degree from the Katholieke Hogeschool Sint-Lieven in Ghent in 2001. After that, he studied Biomedical and Clinical Engineering for which he obtained his M.Sc. degree in 2003. In 2009 he obtained a PhD in engineering from the Ghent University for his work on the topic of Single Photon Emission Computed Tomography. After a one year research stay at the University of Arizona, USA, he became assistant professor at Ghent University. Since then, his research in medical imaging is oriented towards multi-modality imaging, specifically on the integration of Positron Emission Tomography and Single Photon Emission Computed Tomography with Magnetic Resonance Imaging and with a strong emphasis on radiation detector design. His research includes tomographic image reconstruction, medical image processing, objective assessment of image quality and radiation detector and system design.

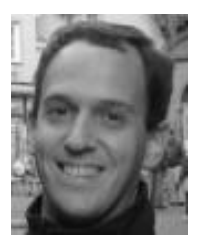

Wout Joseph (M'05) was born in Ostend, Belgium on October 21, 1977. He received the M. Sc. degree in electrical engineering from Ghent University (Belgium), in July 2000.

From September 2000 to March 2005 he was a research assistant at the Department of Information Technology (INTEC) of the same university. During this period, his scientific work was focused on electromagnetic exposure assessment. His research work dealt with measuring and modelling of electromagnetic fields around base stations for mobile communications related to the health effects of the exposure to electromagnetic radiation. This work led to a Ph. D. degree in March 2005.
Since April 2005, he is postdoctoral researcher for iMindsUGent/INTEC. From October 2007 to October 2013, he was a Post-Doctoral Fellow of the FWO-V (Research Foundation Flanders). Since October 2009, he is professor in the domain of Experimental Characterization of wireless communication systems. His professional interests are electromagnetic field exposure assessment, in-body electromagnetic field modelling, electromagnetic medical applications, propagation for wireless communication systems, antennas and calibration. Furthermore, he specializes in wireless performance analysis and Quality of Experience. 